\title{
全球气候变化背景下海岸洪水灾害风险 评估研究进展与展望
}

\author{
方佳毅 ${ }^{1,2,3}$, 史培军 $2,3,4,5^{*}$ \\ (1. 华东师范大学地理科学学院, 地理信息科学教育部重点实验室, 上海 $200241 ; 2$. 北京师范大学地理科学学部, \\ 北京 $100875 ； 3$. 应急管理部-教育部减灾与应急管理研究院,北京 $100875 ；$. 北京师范大学地表过程 \\ 与资源生态国家重点实验室,北京 $100875 ＼mathrm{~ 5 . ~ 青 海 师 范 大 学 地 理 科 学 学 院, 西 宁 ~ 810016) ~}$
}

\begin{abstract}
摘 要:全球气候变化背景下, 海平面上升以及高潮位和风暴潮引起的极值水位导致的海岸洪水对沿海社会经济和 自然环境造成巨大影响, 已是国内外关注的重点。论文梳理了广义和狭义海岸洪水的定义和要素, 重点阐述了狭 义海岸洪水的组成部分, 从致灾因子、孕灾环境和承灾体以及风险评估方法与模型 3 个方面, 系统总结了相关研究 方法与研究成果的主要进展, 以及存在的主要问题, 并透视了未来拟加强的研究方向。建议加强沿海地区应对全 球气候变化风险的研究,包括全球气候变化下多致灾因子耦合危险性和不确定性研究, 沿海关键地区和关键暴露 (关键基础设施)的风险评估研究, 全球气候变化风险适应与减缓性措施的成本效益评价研究, 提高沿海地区应对 全球气候变化风险的韧性研究, 以及建立多学科间的基础数据共享机制, 采用交叉学科手段以便更综合、系统、动 态研究海岸带问题, 保障沿海地区开展全球气候变化下风险评估的需要。
\end{abstract}

关 键 词: 海岸洪水; 全球气候变化; 风暴潮灾害; 风险评估; 影响

全球变暖背景下,海平面上升以及高潮位和风 暴潮引起极值水位导致的海岸洪水对沿海社会经 济和自然环境造成巨大影响。1975-2016年间,全 球 $80 \%$ 因洪水死亡人数在距离沿海 $100 \mathrm{~km}$ 的地区 内 (Hu et al, 2018)。2005 年美国卡特里娜飓风一风 暴潮灾害链使美国新奥尔良市防洪堤决口, 经济损 失达 960 多亿美元(Townsend, 2006)。2008 年纳尔 吉斯热带风暴横扫缅甸三角洲地区, 导致 13.8 万人 失踪死亡(Shibayama, 2015)。此外, 菲律宾的台风 海燕(2013 年)、美国纽约的桑迪飓风(2012 年)和美 国得克萨斯州的哈维飓风(2017年)等都造成巨大的 人员伤亡和经济损失。中国沿海地区是极端天气
和气候事件易发和频发的区域,也是重要的人口与 经济聚集地。随着快速城镇化, 沿海人口急剧膨 胀, 高强度的人类活动、城市化和土地开辱使得中 国沿海土地利用覆盖变化巨大(Liu et al, 2015)。 1989-2014 年间中国因风暴潮引发的海岸洪水灾 害造成约 706 亿美元损失, 失踪死亡约 4354 人 (Fang et al, 2017)。在“一带一路”倡议下, 沿海地区 继续快速发展, 预期未来中国沿海暴露将会持续增 加, 未来沿海地区人口和经济灾害风险仍然趋高。

日益频发的沿海地区极端灾害事件已经引起 了各国政府组织和学术界的高度重视。“未来地球 计划”中确立了海岸带海陆相互作用核心研究项目

收稿日期: 2018-08-03;修订日期:2019-01-15。

基金项目: 国家重点研发计划项目(2016YFA0602404,2017YFE0100700); 上海市科研计划项目(19YF1413700); 博士后科学基 金面上项目 (2019M651429)。[Foundation: National Key Research and Development Program of China, No. 2016YFA0602404 and 2017YFE0100700; Shanghai Sailing Program, No. 19YF1413700; China Postdoctoral Science Foundation, No. 2019M651429. ]

第一作者简介: 方佳毅(1991一),女,浙江金华人,博士后, 助理研究员, 主要从事气候变化及海岸带灾害风险评估研究。

E-mail: jyfang@geo.ecnu.edu.cn

*通信作者简介: 史培军(1959-), 男,陕西靖边人,教授,主要从事环境演变自然灾害与风险管理研究。E-mail: spj@bnu.edu.cn

引用格式: 方佳毅, 史培军. 2019. 全球气候变化背景下海岸洪水灾害风险评估研究进展与展望 [J]. 地理科学进展, 38(5): 625-636. [Fang J Y, Shi P J. 2019. A review of coastal flood risk research under global climate change. Progress in Geography, 38(5): 625-636. ] DOI: $10.18306 /$ dlkxjz.2019.05.001 
(Land-Ocean Interactions in the Coastal Zone, LOICZ), 现已发展为未来地球海岸国际计划(Future EarthCoast, FEC), 旨在全球变化背景下开发和集成多学 科(自然科学+经济+社会)的分析方法, 促进海岸带 地区的可持续发展和提高对气候变化的适应性。 欧盟也就该主题开展了多个大型科研项目(表 1)。 中国关于海岸带和沿海地区全球变化综合风险研 究也实施了一系列重大项目。中国科技部“十三 五”重大科技专项提出, 要求 “评估海岸带和沿海地 区变化综合风险,生成未来 50 100 a 全球变化情景 下, 空间分辨率优于 $1 \mathrm{~km}$ 的中国海岸带及沿海地区 的致灾因子分布图、承灾体脆弱性分布图及综合风 险图”。减轻沿海地区自然灾害风险, 已成为中国 乃至国际社会面临的重大挑战之一。

综上,开展全球气候变化背景下海岸洪水灾害 风险评估研究, 是国际科学研究前沿问题, 对满足
国家及区域发展需要、制定减灾策略、实施海岸带 可持续发展有重大意义。然而, 当前关于海岸洪水 风险的研究,对其定义和成因要素、机制、动力学等 理解不深, 有关概念模糊, 导致研究口径不一致, 影 响研究成果应用等问题。因此,本文从区域灾害系 统的角度出发, 综述国内外海岸洪水相关研究进展 和存在的问题, 为更好地明确研究方向、凝练研究 问题及相关研究提供参考。

\section{1 海岸洪水定义}

当前国内外学术界对海岸洪水(coastal flood)这 一术语的定义还不统一, 可分为广义和狭义 2 种。

广义的海岸洪水(图 1), 指发生在沿海地区的洪 水, 除了海平面上升、天文大潮和风暴潮引起的极 值水位影响, 还可能包括河道型洪水(riverine flood)

表 1 欧盟国家已开展关于气候变化下海岸洪水的部分研究项目信息

Tab.1 Non-exhaustive list of EU funded research projects about coastal flood under climate change

\begin{tabular}{|c|c|c|}
\hline 项目及执行时间 & 项目中英文全称 & 目标 \\
\hline $\begin{array}{l}\text { DINAS-COAST } \\
(2001-2004 \text { 年) }\end{array}$ & $\begin{array}{l}\text { 应对气候变化及海平面上升全球沿海地区动态交互脆弱 } \\
\text { 性评价 (Dynamic and Interactive Assessment of National, } \\
\text { Regional and Global Vulnerability of Coastal Zones to Cli- } \\
\text { mate Change and Sea Level Rise) }\end{array}$ & $\begin{array}{l}\text { 集成多学科的知识(海岸地貌学、生态学、经济学、环境地 } \\
\text { 理和计算机科学),建模开发有助于支持决策者分析的关 } \\
\text { 于气候变化和海平面上升影响的评估模型 }\end{array}$ \\
\hline $\begin{array}{l}\text { XtremRisK } \\
\text { (2008-2012 年) }\end{array}$ & $\begin{array}{l}\text { 极端风暴潮综合洪水风险评估(Integrated Flood Risk Anal- } \\
\text { ysis for Extreme Storm Surges) }\end{array}$ & $\begin{array}{l}\text { 开展在开放海岸和河口地区因极端水位导致的海岸洪水 } \\
\text { 灾害风险分析 }\end{array}$ \\
\hline $\begin{array}{l}\text { THESEUS } \\
\text { (2009-2013 年) }\end{array}$ & $\begin{array}{l}\text { 气候变化下欧洲沿岸创新适应性措施研究(Innovative } \\
\text { Technologies for Safe European Coasts in a Changing Cli- } \\
\text { mate) }\end{array}$ & $\begin{array}{l}\text { 提供针对海岸洪水和海岸侵蚀的综合评估方法,结合多 } \\
\text { 学科知识, 从风险评估、应对策略和应用 } 3 \text { 个具体方向展 } \\
\text { 开,为维护海岸带可持续发展提供创新的适应性措施 }\end{array}$ \\
\hline $\begin{array}{l}\text { RISES-AM } \\
\text { (2013-2016年) }\end{array}$ & $\begin{array}{l}\text { 气候变化下沿海地区应对极端情景创新战略研究—适 } \\
\text { 应与减缓(Responses to Coastal Climate Change: Innovative } \\
\text { Strategies for High End Scenarios - Adaptation and Mitiga- } \\
\text { tion) }\end{array}$ & $\begin{array}{l}\text { 为应对气候变化下沿海地区发生的极端情景, 开发创新 } \\
\text { 性的减轻和适应性措施 }\end{array}$ \\
\hline $\begin{array}{l}\text { SPP } 1889 \\
\text { (2016-2019年) }\end{array}$ & $\begin{array}{l}\text { 区域海平面变化与社会 (Regional Sea Level Change and } \\
\text { Society) }\end{array}$ & $\begin{array}{l}\text { 开展气候相关的海平面变化问题的交叉研究,通过全面、 } \\
\text { 跨学科的集成手段,同时考虑人类环境的交互作用和沿 } \\
\text { 海地区的社会经济发展 }\end{array}$ \\
\hline
\end{tabular}

(a)

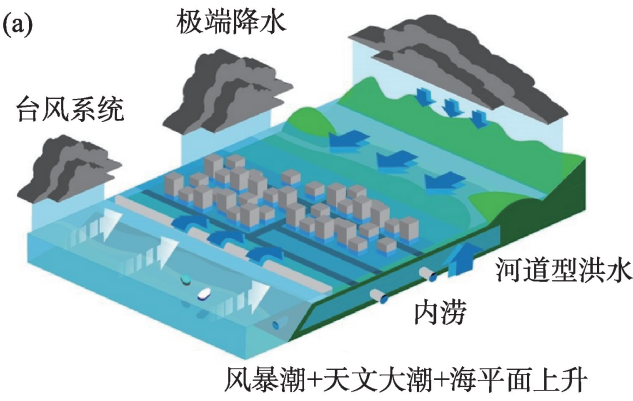

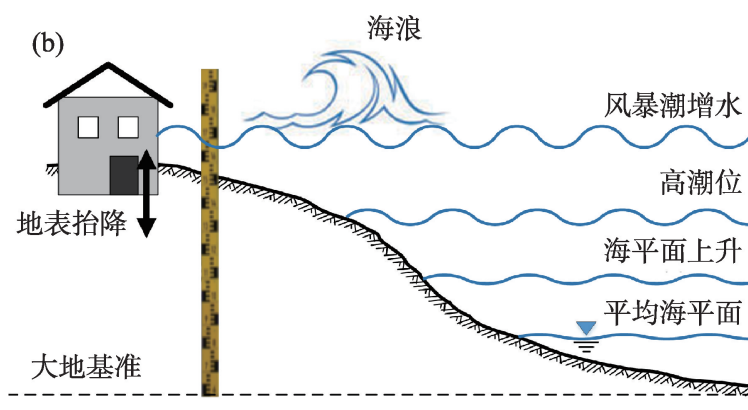

注:广义海岸洪水示意图由美国中佛罗里达大学(University of Central Florida)Thomas Wahl博士提供。

图 1 广义(a)与狭义(b)海岸洪水示意图

Fig.1 Coastal flooding in the broad sense (a) and the narrow sense (b) 
和强降水导致的城市内涝 (waterlogging) 综合作用 的结果, 主要致灾因子可包括超设防陆源洪水、极 端降水和极值海水位。在欧盟 THESEUS 项目中, Zheng 等(2014)和Zscheischler 等(2018)皆采用了广 义海岸洪水概念。广义海岸洪水研究多在河口大 城市区域, 这类地区不仅受到海洋因素的威胁, 还 受到陆源洪水的影响, 叠加极端降水事件, 和复杂 城市系统中高比例的不透水层, 导致城市内涝频 发,上海便是典型地区。

狭义的海岸洪水,一般是指沿海地区气候变化 下海平面上升、风暴潮增水和天文大潮引起的极值 水位导致的洪水。这也是 IPCC 系列报告中常用的 概念, 是基于重现期的极值水位叠加海平面上升进 行淹没结果的分析(IPCC, 2013)。广义海岸洪水研 究涉及多个致灾因子, 研究问题较为复杂。当前大 多数国际研究提及海岸洪水时, 一般指狭义海岸洪 水, 是由于海洋系统不稳定, 如热带气旋风暴潮所 导致的洪水灾害, 水源来自海洋(Nicholls, 2004; Hinkel et al, 2014)。因此本文重点综述气候变化下 狭义海岸洪水风险评估研究进展。

\section{2 海岸洪水致灾因子分析}

狭义海岸洪水所考虑的致灾因子主要可分为 2 部分:代表趋势性的相对海平面变化和代表极端性 的极值水位变化。在 20 世纪 60 年代, 极值水位的 研究主要考虑了因热带气旋引发的风暴潮增水与 高潮位叠加的结果, 开发出一系列风暴潮数值模型 (冯士筰, 1982), 但该阶段对海平面上升的考虑十分 有限。 80 年代以来, 气候变化和海平面上升问题开 始得到重视, 出现了一系列预测未来海平面上升的 研究(IPCC, 1990)。两者研究早期相对独立, 20 世 纪末相关学者意识到, 与海平面缓慢上升引起的静 止水位淹没相比, 叠加极端气候事件引起的极值高 水位导致的灾害后果更为严重, 进而将两者综合考 虑进行研究(Nicholls et al, 2014)。Hoozemans 等 (1993)和 Baarse(1995)初步构建了海平面上升时极 值水位影响的评估方法, 建立了基础数据库, 给出 了全球尺度的影响评估初步结果。Nicholls 等 (1998)、Klein 等(1999)以及 Nicholls(2004)对评估方 法不断改进, 开展了全球范围海岸洪水风险评估研 究, 成为 IPCC 第三、四次报告海岸带章节引用的主 要成果之一。狭义海岸洪水主要的致灾因子组成
部分可由式(1)定义:

$$
\begin{aligned}
\Delta \mathrm{WL}= & \Delta \mathrm{SL}_{\mathrm{G}}+\Delta \mathrm{SL}_{\mathrm{RM}}+\Delta \mathrm{SL}_{\mathrm{RG}}+\Delta \mathrm{SLM}_{\mathrm{H}}+ \\
& \Delta \mathrm{SLM}_{\mathrm{N}}+\eta_{\mathrm{NTR}}+\eta_{\mathrm{W}}
\end{aligned}
$$

式中: $\Delta \mathrm{WL}$ 是总水位的变化; $\Delta \mathrm{SL}_{\mathrm{G}}$ 是全球的平均 海平面变化; $\Delta \mathrm{SL}_{\mathrm{RM}}$ 是区域的由气候海洋因子导致 的海平面差异; $\Delta \mathrm{SL}_{\mathrm{RG}}$ 是区域地球重力场的变化导 致的海平面差异; $\Delta \mathrm{SLM}_{\mathrm{N}}$ 和 $\Delta \mathrm{SLM}_{\mathrm{H}}$ 分别表示自然 和人为因素导致的地表升降变化,其中自然因素的 地表升降包括新构造运动、冰川均衡调整和沉积物 压实/整合, 人为因素导致的地表升降多为地下液体 抽取等因素(Nicholls et al, 2014); 以上 5 个因素通常 为相对海平面变化部分。 $\eta_{\mathrm{NTR}}$ 是风暴潮增水, 中国 学者多把风暴潮增水翻译为 surge 或 storm surge (Kang et al, 2016), 但当前国际研究中, 多用non-tidal residual(NTR)一词来表示风暴潮增水,即总水位 减去天文潮的部分, 天文潮可通过调和分析获得潮 汐变化的部分(Mawdsley et al, 2016); $\eta_{\mathrm{w}}$ 为 波浪增 水,但一般验潮站的观测装置无法捕捉短周期海浪 引起的海水位变化。海啸也会引发极端水位, 但其 引发的极值水位高度远超过了验潮站的测量范围, 通常与地震研究相结合。因此, 在当前海岸洪水研 究中, 大多数研究主要关注天文潮和风暴潮增水的 极值水位组合, 以及全球绝对海平面变化和区域相 对海平面变化。

极值水位的研究主要集中在其历史变化规律 和影响因素, 以及模拟评估极值水位的危险性。国 内外学者对极值水位历史长期变化规律开展了研 究, 发现全球大部分验潮站的极值水位呈现增加趋 势, 但将极值水位减去平均海平面变化后发现这种 增加趋势大幅减少, 由此认为极值水位的大部分变 化是由于海平面变化引起的 (Woodworth et al, 2004; Marcos et al, 2009; Menéndez et al, 2010)。也 有研究指出极值水位增加速度明显高于平均海平 面增长(Woodworth et al, 2011)。中国沿海极值水位 呈现显著增长趋势, 在 1954-2012 年间, 以2.0 $14.1 \mathrm{~mm} / \mathrm{a}$ 速率增加(Feng et al, 2014; Feng et al, 2015)。海平面变化除了在极值水位长期变化中起 到重要作用, 也会导致极值水位频率增加。早期研 究表明, 极值水位重现期会因海平面的轻微变化有 巨大改变(Nicholls et al, 1999)。其他国内外研究也 得到类似结论(施雅风等, 1994; 施雅风等, 2000; Wu et al, 2017)。极值水位除受到海平面变化的影 
响外, 还受到台风、风暴潮、风场和大气环流指数等 因素的影响(Wahl, Chambers, 2015)。除了研究极值 水位的历史变化规律和其影响因素, 也有大量研究 评估极值水位的危险性。极值水位危险性评估是 风险评估研究的基础, 主要通过 2 种方法, 即统计模 型和数值模拟。国际上已建立了较为成熟的风暴 潮数值模型，如 SLOSH、ADCIRC、DELFT3D 、 MIKE21 和 GCOM 2D/3D 模型等(石先武等, 2013)。荷兰学者基于水动力模型开发了全球第一 套风暴潮和极值水位数据集(Muis et al, 2016)。目 前针对中国沿海地区极值水位的研究, 由于沿海站 点分布较为稀疏且时间序列较短, 多以验潮站的站 点数据计算不同重现期下风暴潮增水高度(Wahl et al, 2017; Wu et al, 2017; 李阔等, 2017), 在此基础上 开展风险评估工作。

海平面变化常被划分为因海洋水体总体质量 变化和比容效应导致的绝对海平面变化和相对某 一基准面(如大地基准或历史平均海平面)的相对海 平面变化(陈美香等, 2013)。预测未来海平面变化 趋势的常用方法分 2 类: 一是利用奇异谱分析、灰色 模型、经验模态法、自回归模型、小波分析等统计模 型方法对历史观测数据进行分析, 找出其规律进行 外推预测(段晓峰等, 2014); 二是使用全球耦合模式 对未来不同温室气体排放情景下, 对海平面变化进 行模拟分析(左军成等, 2015)。利用统计模型的外 推来预测未来变化, 受数据序列长度和质量的影响 较大, 且假设未来海平面变化系统处于稳定状态, 其变化规律保持不变, 因此不能反映因气候变化导 致的海洋系统变化的实际情况。相比而言, 基于大 型数值模式的全球海平面变化研究是当前研究的 主流, 也是 IPCC 系列报告采用的主要方法(Church et al, 2013; Jevrejeva et al, 2014; Kopp et al, 2014)。
在 IPCC AR5 报告中, 基于 CMIP5 全球气候模式对 未来多种排放情景进行了模拟(温家洪等, 2018)。 当前耦合数值模型存在对全球气候变化过程模拟 刻画得不够完善、模型中不确定因素较多且模式的 精度不够高等问题,全球大量的学者在试图改进模 型模拟的物理机制, 提高海平面上升数据产品的分 辨率, 使得模拟过程更合理, 数据产品质量和精度 更高。

表 2 给出了已有(狭义)海岸洪水风险评估的相 关案例及其考虑的因素。大部分研究考虑平均海 平面的变化、天文大潮和风暴潮增水叠加作用,但 由于海浪观测数据较少且模拟的技术难度较大, 因 而在相关风险评估中考虑较少; 对于区域海平面差 异考虑也较少; 对自然因素的地面升降考虑较多, 但对人为因素导致地面升降考虑较少。值得注意 的是, 以上研究是将海平面上升和极值水位进行线 性叠加, 未考虑两者耦合机制。Lin 等(2012)就这一 问题的研究有了突破,基于物理机制, 用数值模型 分析海平面上升情景下纽约地区风暴潮水位的变 化。但由于研究涉及大量数值运算, 难度较大, 需 要多学科、多单位的合作努力, 相关研究成果较 少。因此当前相关研究中, 大多依然对海平面上升 和极值水位进行线性叠加。

\section{3 孕灾环境和承灾体研究}

地形地貌、海岸工程和地面沉降等自然和人文 孕灾环境,对沿海地区海岸洪水致灾-成害过程有 重要影响。随着研究深人, 近年来学者们越来越重 视实际设防水平和地面沉降在海岸洪水致灾过程 中的影响(Ward et al, 2017)。关于沿海设防水平的 数据十分有限, 当前国内外相关评估研究根据人均

表 2 已有海岸洪水风险评估的相关案例

Tab.2 Non-exhaustive examples of coastal flooding assessment

\begin{tabular}{|c|c|c|c|c|c|c|c|}
\hline \multirow{2}{*}{ 文献 } & \multirow{2}{*}{ 内容 } & \multicolumn{6}{|c|}{ 海平面组成 } \\
\hline & & $\triangle \mathrm{SL}_{\mathrm{G}}$ & $\triangle \mathrm{SL}_{\mathrm{RM}}$ & $\triangle \mathrm{SL}_{\mathrm{RG}}$ & $\triangle \mathrm{SLM}_{\mathrm{N}}$ & $\triangle \mathrm{SLM}_{\mathrm{H}}$ & $\triangle \mathrm{SS}$ \\
\hline Lowe et al, 2009 & 国家层面(英国) & V & V & & V & & V \\
\hline Rosenzweig, 2010 & 城市(纽约) & V & & & V & & V \\
\hline Hanson et al, 2011 & 全球(城市) & V & & & V & V & V \\
\hline Parris et al, 2012 & 国家层面(美国) & V & V & & V & & V \\
\hline Wang et al, 2012 & 城市(中国上海) & $\checkmark$ & & & V & V & V \\
\hline Kebede et al, 2012 & 城市(坦桑尼亚首都) & $\checkmark$ & & & V & & V \\
\hline
\end{tabular}

注: $\triangle \mathrm{SS}$ 为相对于平均海平面极值水位的部分。 
GDP来简化设防水平, 或直接不考虑设防(Aerts et $a l, 2016)$ 。但在实际情况中, 在人口聚集地和重大 沿海城市都有一定程度的设防。在中国沿海地区， 为了抵御海岸侵蚀、海岸洪水和灾难性海浪等沿海 极端气象海洋灾害事件,已建立了一系列防护措施 来保护沿海的人口安全和经济活动, 研究表明全国 超过 60\%的海岸线已受到海堤保护(Cai et al, 2009; Ma et al, 2014)。若不考虑设防, 会使得其风险评估 结果偏高。此外, 全球沿海三角洲和重大城市存在 严重的地面沉降问题(Syvitski et al, 2009), 使得地 面高程降低, 相对海平面上升加快, 进而降低了设 防能力, 因此在风险评估中越来越重视自然因素和 人为因素导致的地面升降影响(Woodruff et al, 2013)。已有研究将这一孕灾环境考虑在致灾因子 危险性中,指出地面沉降改变了相对海平面上升高 度, 放大了危险性(Nicholls et al, 2010)。在中国沿 海大城市, 人为因素导致的地面沉降已十分严重 (郑铣金等, 2001), 但目前有关风险评估中考虑地面 沉降的研究十分有限。

近年来国内外学者针对人口、经济、农业和湿 地等承灾体, 评估了海平面变化下极值水位对社会 经济和自然环境的影响。Nicholls(2004)和 Spencer 等(2016)评估了海岸洪水的影响和湿地损失; Hanson 等(2011)评估了全球 136 个港口城市未来在百 年一遇海岸洪水事件下的人口和资产风险; Jongman 等(2012)计算了在百年一遇河道型和海岸洪水 事件下的 2010 年和 2050 年资产暴露度; Hallegatte 等(2013)评估了 136 个沿海主要城市在未来海岸洪 水下的暴露、损失和风险, 并对其进行了风险排名; Hinkel 等(2014)分析了未来全球海岸洪水下的人口 与资产风险, 强调了适应性措施的重要性; Vousdoukas 等(2018)分析了多种气候情景和社会经济情 景对海岸洪水风险变化的贡献率。承灾体并非静 止不变, 而是在动态变化, 如未来老龄人口将不断 增多, 沿海城市的流动性人口存在季节性特点, 土 地利用类型在不断变化, 沿海暴露可能不断增多。 虽然已有结合未来多种气候情景和社会经济情景 的风险评估, 但此类研究多集中于人口和经济总量 方面, 如 Hinkel 等(2014) 和 Vousdoukas 等(2018), 且 其他承灾体的动态研究十分有限。另外, 对影响灾 害损失的关键暴露的研究较少, 特别是包括电力设 施、交通枢纽、避难所、物资储备基地、水闸和提防 设施等在内的沿海重要关键基础设施(Brown et al,
2014; Willis et al, 2016)。关键基础设施的破坏会导 致区域基础设施功能的系统性、级联性痽疾, 进而 引发其他间接损失, 而目前间接损失的评估和对系 统动态网络功能影响评估的研究还较少。

承灾体脆弱性指社会经济系统和生态系统遭 受到致灾因子打击的可能性大小,最常见的是物理 脆弱性和社会脆弱性(UNISDR, 2009)。承灾体物 理脆弱性侧重于致灾因子物理特性和承灾体的响 应。物理脆弱性分析采用定量分析的手段,基于灾 情数据、现场调研数据、保险数据、模型模拟等手 段, 获取损失与致灾因子之间的关系, 给出定量风 险评估结果(周瑶等, 2012)。通过构建淹没水深与 沿岸承灾体(人口、房屋、海堤等)损失之间的函数关 系, 可以构造脆弱性损失矩阵或建立脆弱性曲线来 确定不同致灾强度作用下承灾体的损失率(石先武 等, 2016)。国外对洪水灾害脆弱性曲线开展了大 量的研究,如美国、英国和荷兰已针对不同建筑物 类型建立损失曲线(FEMA, 2015; Jonkman et al, 2008)。国内在上海等部分地区也开展了较为详细 的研究(尹占娥等, 2012)。因物理脆弱性研究对历 史灾情数据要求较高, 需要大量的实地调研, 而当 前灾情数据公开较少或质量不高, 资料获取困难, 水深和损失之间的脆弱性曲线研究十分有限, 难以 构建出普适、实用的物理脆弱性曲线,使得在目前 研究中较多依赖于国际的物理脆弱性曲线。社会 脆弱性可以理解为社会系统遭遇致灾事件冲击的 敏感性, 以及应对灾害事件的适应能力(Cutter et al, 2008)。评价方法通常采用指标体系法, 通过建立 指标评价体系, 用专家打分法、因子分析法等手段 给因子赋予权重, 对社会脆弱性等级进行划分。利 用这些方法, 就中国沿海地区不同尺度和不同研究 区的社会脆弱性评价已有一定进展, 如对沿海市级 (谭丽荣, 2012; Su et al, 2015)和沿海县级为单元(方 佳毅等, 2015)开展了社会脆弱性评价。但评价指 标选取和建立, 权重赋予方法各不相同, 对结果影 响较大, 主观性较大。此外, 指标体系法得到的是 无单位的标量或相对值, 可以用于识别高脆弱性区 域,或脆弱性变化趋势, 但它与损失之间的定量关 系还不清晰, 因此较难应用于定量风险评估中。

针对海岸带系统, 大量学者也开展了海岸带承 灾体综合脆弱性和易损性方面的研究, 建立了脆弱 性评估模型, 如 PSR 模型(Pressure-State-ResponseFramework)、DPSIR 模型 (Driving force- Pressure- 
State- Impact- Response) 和 SPRC(Source- PathwaysReceptor-Consequence) 等(王宁等, 2012; 王腾等, 2015)。基于以上概念模型, 相关学者建立了海岸 带综合脆弱性评级模型, 一般是指标体系法(李恒 鹏等, 2002; 储金龙等, 2005; 孙蕾等, 2007; Yin et $\mathrm{al}, 2013$; 李响等, 2016), 但以上研究所得的脆弱性 也是标量结果, 较难与定量风险评估联系起来。

\section{4 海岸洪水风险评估方法和模型}

在确定了海岸洪水需要考虑的要素后, 如何确 定极值水位下的海岸洪水淹没范围成为风险分析 中最关键的问题(Mcleod et al, 2010)。目前确定海 岸洪水淹没范围的手段主要可以总结为 2 类:一类 是基于 GIS 支持下的高程-面积法, 二是基于水动 力演化的数值模型。

当前研究使用最普遍的方法是基于 GIS 手段 的评估模型, 特别在大尺度海岸洪水研究中有广泛 应用(Hall et al, 2003; Rowley et al, 2007; Fang et al, 2014)。但该类方法的缺陷是没有考虑极值水位的 持续时间, 未考虑地面粗粘度,并非某特定水位下 的区域都是受灾区, 容易高估风险; 优点是能较快 划分出高风险区域, 特别是针对未来多种气候情景 下的影响, 可以为全球和国家相关决策者提供海岸 带宏观灾害风险防范的信息。

基于水动力演化的数值模型, 如 ADCIRC、 DELFD3D 等大型风暴潮数值模型, 能够较好地模 拟风暴潮增水和漫滩等过程, 但应用于大尺度海岸 洪水灾害风险评估中却较难实现, 主要原因是 : (1) 模型所需数据庞大复杂; (2) 求解过程复杂耗时; (3) 对致灾因子强度有较好的模拟, 但对风险评估中承
灾体脆弱性等其他因素考虑不足。随着地形数据 精度的提高, 例如 $5 \mathrm{~m}$ 及 $5 \mathrm{~m}$ 以下的 LiDAR 高程数 据,使得基于 GIS 栅格数据二维洪水模型的使用更 为广泛, 例如 Lisflood(Bates et al, 2000)、JFLOW (Bradbrook, 2006) 和 Floodmap(Yu et al, 2006a; 2006b)等。该类二维洪水模型简化了物理过程, 对 求解效率有很大的提高, 对小尺度研究有很好的表 现(Yin et al, 2016)。为了提高极值水位模拟,简化 求解过程, 提高效率, 一些学者采用其他研究团队 开发的相关风暴潮产品,一般是基于大型风暴潮数 值模式且针对某些特定区域的产品,如多种重现期 下的极值水位高度, 作为陆地上漫滩过程的输人, 再使用基于 GIS 栅格数据的二维洪水模型作为洪 水过程的演进(例如Yin et al, 2017)。但该方法目前 也较难应用于大尺度海岸洪水风险评估中, 主要由 于对基础数据精度要求极高, 而且就大尺度而言, 基础数据量庞大也较难获得。

综上所述, 在大尺度, 如全球尺度或国家层面 上,开展全球气候变化下海岸洪水对沿海地区的影 响评估中更多依赖于基于 GIS 模型的高程面积 法。除了普遍的 GIS 手段的评估模型,表 3 总结了 目前已有的海岸洪水影响评估模型及其主要参 数。这类评估模型集成了多学科知识, 一定程度上 考虑了自然环境和社会经济环境的动态反馈, 可为 决策者和利益相关者提供更有效的信息, 提高了评 估效率。

\section{5 国内相关研究中存在的问题}

通过国内外研究, 本文发现欧美发达国家在多 种气候情景下的海岸洪水灾害风险评估研究已较

表 3 海岸洪水影响评估模型及其主要参数

Tab.3 Key attributes of coastal flood impact models

\begin{tabular}{|c|c|c|c|c|c|c|}
\hline 模型 & 尺度 & 空间分辨率 & 时间尺度 & 输人数据 & 输出数据 & 文献 \\
\hline 淹没模型(如 GIS) & $\begin{array}{l}\text { 地方、区域 } \\
\text { 和全球 }\end{array}$ & 多变 & 用户定义 & $\begin{array}{l}\text { 高程、海平面上升情 } \\
\text { 景、社会经济数据 }\end{array}$ & $\begin{array}{l}\text { 潜在淹没地区和影响 } \\
\text { 人口地图 }\end{array}$ & Rowley et al, 2007 \\
\hline $\begin{array}{l}\text { SLAMM (Sea Level Af- } \\
\text { fecting Marshes Model) }\end{array}$ & 地方和区域 & $10 \sim 100 \mathrm{~m}$ & $\begin{array}{l}5 \sim 25 \mathrm{a} \text { 时 } \\
\text { 间步长 }\end{array}$ & $\begin{array}{l}\text { 高程地图、湿地覆盖、 } \\
\text { 发展足迹和海堤位置 }\end{array}$ & $\begin{array}{l}\text { 潜在淹没地区和影响 } \\
\text { 人口地图 }\end{array}$ & Galbraith et al, 2003 \\
\hline $\begin{array}{l}\text { DIVA (Dynamic Interac- } \\
\text { tive Vulnerability Assess- } \\
\text { ment) }\end{array}$ & $\begin{array}{l}\text { 国家、区域、 } \\
\text { 全球 }\end{array}$ & $\begin{array}{l}\text { 海岸线分段(全球 } \\
\text { 分为 } 1.2 \text { 万段,平 } \\
\text { 均每段 } 70 \mathrm{~km})\end{array}$ & $\begin{array}{l}1 \sim 5 \mathrm{a}, \text { 长 } \\
\text { 达 } 100 \mathrm{a}\end{array}$ & $\begin{array}{l}\text { 高程、地貌类型、海岸 } \\
\text { 人口、土地利用、行政 } \\
\text { 边界、GDP }\end{array}$ & $\begin{array}{l}\text { 预计海岸洪水影响人 } \\
\text { 口、湿地改变、损失和 } \\
\text { 适应成本、土地损失 }\end{array}$ & Hinkel et al, 2009 \\
\hline $\begin{array}{l}\text { LISCoAsT (Large scale } \\
\text { Integrated Sea- level and } \\
\text { Coastal Assessment Tool) }\end{array}$ & 欧洲区域 & $\begin{array}{l}\text { 海岸线分段, 长 } \\
\text { 度不等 }\end{array}$ & $\begin{array}{l}\text { 多变, 用户 } \\
\text { 定义 }\end{array}$ & $\begin{array}{l}\text { 高程、气象数据、人口 } \\
\text { 等 }\end{array}$ & $\begin{array}{l}\text { 期望人口与经济损失 } \\
\text { 等 }\end{array}$ & $\begin{array}{l}\text { Vousdoukas et al, } \\
2018\end{array}$ \\
\hline
\end{tabular}

注:修改自 Mcleod等(2010)。 
为深人,已开展了国家层面的海岸洪水风险评估工 作, 如德国(Sterr, 2008)、美国(Parris et al, 2012)、加 拿大(Lemmen et al, 2016)和英国(Sayers et al, 2017) 等, 但中国尚未有国家层面上的相关评估报告。相 比于国外, 国内关于海平面上升和极值水位叠加的 海岸洪水相关研究起步较晚, 且目前还大多针对致 灾因子的危险性, 对未来气候情景下区域尺度的海 平面上升的预估研究较多, 而对未来不同气候情景 下, 中国沿海地区的灾害风险评估较少。国内已有 的相关研究大多针对某一区域, 如珠三角地区 (Kang et al, 2016)和上海(Wang et al, 2012), 在国家 层面上开展海平面上升和极值水位叠加的海岸洪 水风险评估工作十分有限。与此同时, 国内相关自 主开发并公开的数据产品较少, 社会经济数据、灾 情数据存在统计口径不统一、年份缺失较多的情 况,成为当前研究的一大瓶颈。

(1) 缺少致灾因子耦合危险性研究。大多相关 研究中假定平均海平面上升和导致极值水位的风 暴潮系统相对独立, 两者之间是线性叠加的关系, 并假定系统是稳定的, 未考虑全球气候变化对风暴 潮整体系统的改变, 也未考虑区域波动性。全球气 候变化可能会使得海洋系统整体发生相应变化, 需 要警惕极端情景(high-end scenario)和“灰天鹅”事件 (Grey Swan) 的出现 (Lin et al, 2016; Rahmstorf, 2017), 注意非一致性和平稳性过程。在海岸河口 地区, 由于近岸地形作用和多种水源叠加, 容易出 现多灾种叠加问题, 可能会使得致灾程度高于仅考 虑单一极值海水位的影响, 是目前国际关注的重点 (Zscheischler et al, 2018)。国外已开展用联合概率 分布研究多种海岸洪水致灾因子叠加的非线性效 应, 如将河流径流洪水与海岸洪水结合 (Lamb et al, 2010), 或将海岸洪水与极端降水结合(Wahl, Jain et $\mathrm{al}, 2015)$ 。目前国内相关研究较为薄弱, 是未来突 破的难点之一。

(2) 缺乏学科间的融合, 缺少对孕灾环境和人 为因素的考虑。从灾害系统的角度理解, 海岸洪水 灾害风险变化受到自然和人类社会多方面要素的 影响, 但目前评估研究中考虑的要素较少, 并且缺 少考虑多情景和人为要素。虽然各领域专家分别 从海洋学、地质学和地理学的角度进行了研究, 但 缺乏融合各学科的系统研究(Hinkel et al, 2015)。 目前中国相关研究大多是从全球气候变化角度开 展的工作, 通常基于某一种气候模式或者某种排放 情景开展海岸灾害风险评估, 缺少对陆面系统及人
类经济系统的考虑, 在评估中考虑沿海设防和地面 沉降控制等因素十分有限, 同时考虑未来气候情景 变化和社会经济情景变化这两者因素的研究更 少。虽然已有分别对中国未来人口和经济的模拟 研究(姜軕等, 2017; 姜制等, 2018), 但目前评估研究 中还未将两者结合。

(3) 沿海地区适应性措施研究和韧性研究较为 欠缺。用定量化的成本-效益分析的手段,开展评 估各种海岸工程或非工程的适应性与减缓性措施, 以预防、应对或减缓气候变化和灾害风险是当前的 流行研究趋势(Aerts et al, 2014; Ward et al, 2017)。 沿海地区在应对全球气候变化的适应性措施除了 设防, 还有其他措施, 可总结为防护(protection)、后 退(retreat) 和顺应(accommodation) 3 类; 按工程性 质, 可分为工程性措施和非工程性措施(Linham et al, 2012)。但目前中国沿海地区适应性措施研究处 于初步阶段(冯爱青等, 2016)。另外大多数研究开 始从脆弱性视角向韧性(Resilience)视角转变, 韧性 在越来越多的海岸带研究中得到关注(Aerts et al, 2014)。韧性研究是一个多学科框架下探索系统内 部的应激、恢复、适应以及转型能力, 强调系统对外 界干扰的自主抵抗(Pelling et al, 2014)。但目前关 注中国沿海地区韧性的研究也十分有限。

\section{6 展望}

随着中国沿海地区经济的持续发展, 以及“一 带一路”倡议下, 需要加强沿海地区应对全球气候 变化风险的研究。基于以上不足, 本文提出了以下 几点展望。

（1）加强气候变化下多致灾因子耦合危险性和 不确定性研究。未来需加强全球海平面上升对热 带气旋系统的影响和海岸带系统的交互影响,基于 物理机制, 用数值模型模拟全球海平面上升下热带 气旋系统和极值水位的变化。加强对灾害链和灾 害群的致灾机制研究,用统计模型或动力模型分析 多致灾因子耦合的非线性效应。加强致灾因子不 确定性研究, 用随机模拟生成大量台风路径和强度 的随机数据集, 利用数值模式计算并分析模拟中不 确定性。在海岸致灾因子危险性深化研究的基础 上,自主开发针对整个中国沿海的致灾因子产品和 相关评估软件, 强化自主模型研究, 更好地为海洋 工程服务, 为利益相关者提供信息。

(2) 加强对沿海关键地区和关键暴露(关键基础 
设施)的风险评估研究。未来应当抓紧开展对沿海 关键地区和关键暴露的调查与隐患排查工作, 重点 调查高脆弱人群(如老龄人口和流动人口), 并对可 能造成重大影响的关键基础设施(如堤坝、电力设 施、交通枢纽)进行排查。可选择典型区, 尝试对未 来承灾体的预测研究, 针对不同土地利用类型和基 础设施等关键暴露建立相应的脆弱性曲线, 开展综 合人口与经济风险评估工作。

(3) 加强全球气候变化风险适应与减缓性措施 的成本效益评价研究。目前多种适应性和减缓性 措施对海岸带环境的影响研究十分有限, 特别是堤 坝建立、土地围层等措施。未来应加强在全球气候 变化背景下设防、围填海等工程性措施对海岸带环 境综合影响的研究。对于海岸带系统而言, 面对未 来全球气候变化和极端灾害事件时, 如何提高沿海 地区韧性, 更好地适应全球变化, 这些研究将显 得越来越重要。

(4) 加强数据开放力度与多学科的交叉研究。 建议有关部门加强对用于科研项目所需数据的开 放力度, 加强对基础社会经济数据的收集和统计, 制定统计规范标准,建立更有效的社会信息收集系 统和更为完备的数据资料库。随着网络技术的快 速发展, 利用大数据进行研究也是未来一大趋势。 未来应加强多学科之间的基础数据共享机制, 采用 交叉学科手段, 将其他学科(如经济学、社会学、系 统动力学) 的新兴手段应用到沿海地区, 以便更综 合、系统、动态地研究海岸带问题。

\section{参考文献(References)}

陈美香, 白如冰, 左军成, 等. 2013. 我国沿海海平面变化预 测方法探究 [J]. 海洋环境科学, 32(3): 451-455. [Chen M X, Bai R B, Zuo J C, et al. 2013. Discussion of sea level prediction along the coastal of China. Marine Environmental Science, 32(3): 451-455. ]

储金龙, 高抒, 徐建刚. 2005. 海岸带脆弱性评估方法研究进 展 [J]. 海洋通报, 24(3): 80-87. [Chu J L, Gao S, Xu J G. 2005. Risk and safety evaluation methodologies for coastal systems: A review. Marine Science Bulletin, 24(3): 80-87. ] 段晓峰, 许学工, 陈满春, 等. 2014. 基于长期验潮数据的海 平面预测方法与案例应用 [J]. 北京大学学报(自然科学 版), 50(6):1065-1070. [Duan X F, Xu X G, Chen M C, et al. 2014. Methodology and case study of sea level prediction based on secular tide gauge data. Acta Scientiarum Naturalium Universitatis Pekinensis, 50(6): 1065-1070. ] 方佳毅, 陈文方, 孔锋, 等. 2015. 中国沿海地区社会脆弱性
评价 [J]. 北京师范大学学报 (自然科学版), 51(3): 280286. [Fang J Y, Chen W F, Kong F, et al. 2015. Measuring social vulnerability to natural hazards of the coastal areas in China. Journal of Beijing Normal University (Natural Science), 51(3): 280-286. ]

冯爱青, 高江波, 吴绍洪, 等. 2016. 气候变化背景下中国风 暴潮灾害风险及适应对策研究进展 [J]. 地理科学进展, 35(11): 1411- 1419. [Feng A Q, Gao J B, Wu S H, et al. 2016. A review of storm surge disaster risk research and adaptation in China under climate change. Progress in Geography, 35(11): 1411-1419. ]

冯士筰. 1982. 风暴潮导论 [M]. 北京: 科学出版社. [Feng S Z. 1982. Introduction to storm surge. Beijing, China: Science Press. ]

姜形, 赵晶, 曹丽格, 等. 2018. 共享社会经济路径下中国及 分省经济变化预测 [J]. 气候变化研究进展, 14(1): 50-58. [Jiang T, Zhao J, Cao L G, et al. 2018. Projection of national and provincial economy under the shared socioeconomic pathways in China. Climate Change Research, 14(1): 50-58. ] 姜粀, 赵晶, 景丞, 等. 2017. IPCC 共享社会经济路径下中国 和分省人口变化预估 [J]. 气候变化研究进展, 13(2): 128137. [Jiang T, Zhao J, Jing C, et al. 2017. National and provincial population projected to 2100 under the shared socioeconomic pathways in China. Climate Change Research, 13(2): 128-137. ]

李恒鹏, 杨桂山. 2002. 全球环境变化海岸易损性研究综述 [J]. 地球科学进展, 17(1): 104-109. [Li H P, Yang G S. 2002. The advance in studies on coastal vulnerability to global change. Advance in Earth Sciences, 17(1): 104-109. ] 李阔, 李国胜. 2017. 气候变化影响下 2050 年广东沿海地区 风暴潮风险评估 [J]. 科技导报, 35(5): 89-95. [ $\mathrm{Li} \mathrm{K}, \mathrm{Li} \mathrm{G}$ S, 2017. Risk assessment of storm surges in the coastal area of Guangdong Province in year 2050 under climate change. Science \& Technology Review, 35(5): 89-95. ]

李响, 段晓峰, 张增健, 等. 2016. 中国沿海地区海平面上升 脆弱性区划 [J]. 灾害学, 31(4): 103-109. [Li X, Duan X F, Zhang Z J, et al. 2016. The vulnerability zoning research on the sea-level rise of Chinese Coastal. Journal of Catastrophology, 31(4): 103-109. ]

施雅风, 杨桂山. 1994. 中国海平面上升及其影响评估: 海平 面上升对中国三角洲地区的影响及对策 [M]. 北京: 科学 出版社. [Shi Y F, Yang G. 1994. Sea level rise and its impacts in China: Impacts and countermeasures of sea level rise on China's delta region. Beijing, China: Science Press. ] 施雅风, 朱季文, 谢志仁, 等. 2000. 长江三角洲及毗连地区 海平面上升影响预测与防治对策 [J]. 中国科学: 地球科 学, 30(3): 225-232. [Shi Y F, Zhu J W, Xie Z R, et al. 2000. Prediction and countermeasures of sea level rise in 
the Yangtze River Delta and adjacent areas. Science in China: Earth Sciences, 30(3): 225-232. ]

石先武, 国志兴, 张尧, 等. 2016. 风暴潮灾害脆弱性研究综 述 [J]. 地理科学进展, 35(7): 889-897. [Shi X W, Guo Z $\mathrm{X}$, Zhang Y, et al. 2016. A review of research on vulnerability to storm surges. Progress in Geography, 35(7): 889-897. ] 石先武, 谭骏, 国志兴, 等. 2013. 风暴潮灾害风险评估研究 综述 [J]. 地球科学进展, 28(8): 866-874. [Shi X W, Tan J, Guo Z X, et al. 2013. A review of risk assessment of storm surge disaster. Advances in Earth Science, 28(8): 866-874. ] 孙蕾, 石纯. 2007. 沿海城市自然灾害脆弱性评估研究进展 [J]. 灾害学, 22(1): 102-105. [Sun L, Shi C. 2007. Progress in vulnerability assessment of natural disasters in coastal cities. Journal of Catastrophology, 22(1): 102-105. ]

谭丽荣. 2012. 中国沿海地区风暴潮灾害综合脆弱性评估 [D]. 上海: 华东师范大学. [Tan L R. 2012. Assessment on comprehensive vulnerability of storm surge disasters of China's coastal regions. Shanghai, China: East China Normal University. ]

王宁, 张利权, 袁琳, 等. 2012. 气候变化影响下海岸带脆弱 性评估研究进展 [J]. 生态学报, 32(7): 2248-2258. [Wang N, Zhang L Q, Yuan L, et al. 2012. Research into vulnerability assessment for coastal zones in the context of climate change. Acta Ecologica Sinica, 32(7): 2248-2258. ] 王腾, 邹欣庆, 李保杰. 2015. 多驱动因素下海岸带脆弱性研 究进展 [J]. 海洋通报, 34(4): 361-369. [Wang T, Zou X Q, Li B J. 2015. Research progress of coastal vulnerability to varied driving factors. Marine Science Bulletin, 34(4): 361369. ]

温家洪, 袁穗萍, 李大力, 等. 2018. 海平面上升及其风险管 理 [J]. 地球科学进展, 33(4): 350-360. [Wen J H, Yuan S $\mathrm{P}$, Li D L, et al. 2018. Sea level rise and its risk management. Advances in Earth Science, 33(4): 350-360. ]

尹占娥, 许世远. 2012. 城市自然灾害风险评估研究 [M]. 北 京: 科学出版社. [Yin Z E, Xu S Y. 2012. Study on risk assessment of urban natural hazards. Beijing, China: Science Press. ]

郑铣金金, 武强, 应玉飞, 等. 2001. 中国沿海地区相对海平面 上升的影响及地面沉降防治策略 [J]. 科技通报, 17(6): 51-55. [Zheng X X, Wu Q, Ying Y F, et al. 2001. Impacts of relative sea-level rising and strategies of control of land subsidence in coastal region of China. Bulletin of Science and Technology, 17(6): 51-55. ]

周瑶, 王静爱. 2012. 自然灾害脆弱性曲线研究进展 [J]. 地球 科学进展, 27(4): 435-442. [Zhou Y, Wang J A. 2012. A review on development of vulnerability curve of natural disaster. Advances in Earth Science, 27(4): 435-442. ]

左军成, 左常圣, 李娟, 等. 2015. 近十年我国海平面变化研
究进展 $[\mathrm{J}]$. 河海大学学报(自然科学版), 43(5): 442-449. [Zuo J C, Zuo C S, Li J, et al. 2015. Advances in research on sea level variations in China from 2006 to 2015. Journal of Hohai University (Natural Sciences), 43(5): 442-449. ]

Aerts J C J H, Botzen W J W, Emanuel K, et al. 2014. Evaluating flood resilience strategies for coastal megacities $[\mathrm{J}]$. Science, 344: 473-475.

Aerts J C J H, Bouwer L M, Winsemius H C, et al. 2016. FLOPROS: An evolving global database of flood protection standards [J]. Natural Hazards and Earth System Sciences, 16(5): 1049-1061.

Baarse G. 1995. Development of an operational tool for Global Vulnerability Assessment (GVA): Update of the number of people at risk due to sea level rise and increasing flooding probability $[\mathrm{M}]$. CZM- Centre Publication No. 3. Hague, the Netherlands: Ministry of Transport, Public Works and Water Management.

Bates P D, De Roo A P J. 2000. A simple raster-based model for flood inundation simulation [J]. Journal of Hydrology, 236(1): 54-77.

Bradbrook K. 2006. JFLOW: A multiscale two-dimensional dynamic flood model [J]. Water and Environment Journal, 20(2): 79-86.

Brown S, Hanson S, Nicholls R J. 2014. Implications of sealevel rise and extreme events around Europe: A review of coastal energy infrastructure [J]. Climatic Change, 122(12): 81-95.

Cai F, Su X, Liu J, et al. 2009. Coastal erosion in China under the condition of global climate change and measures for its prevention [J]. Progress in Natural Science, 19(4): 415-426.

Church J A, Clark P U, Cazenave A, et al. 2013. Sea-level rise by 2100 [J]. Science, 342: 1445. doi: 10.1126/science.342. 6165.1445-a.

Cutter S L, Finch C. 2008. Temporal and spatial changes in social vulnerability to natural hazards [J]. PNAS, 105(7): 2301-2306.

Fang J, Liu W, Yang S, et al. 2017. Spatial-temporal changes of coastal and marine disasters risks and impacts in Mainland China [J]. Ocean \& Coastal Management, 139: 125-140.

Fang J, Sun S, Shi P, et al. 2014. Assessment and mapping of potential storm surge impacts on global population and economy $[\mathrm{J}]$. International Journal of Disaster Risk Science, 5(4): 323-331.

FEMA. 2015. HAZUS- MH flood model: Technical manual [EB/OL]. 2015-12-01[2017-08-22]. https://www.fema.gov/ media-library/assets/documents $/ 24609$ ? id $=5120$.

Feng J, von Storch H, Jiang W, et al. 2015. Assessing changes in extreme sea levels along the coast of China $[\mathrm{J}]$. Journal 
of Geophysical Research: Oceans, 120(12): 8039-8051.

Feng X, Tsimplis M N. 2014. Sea level extremes at the coasts of China [J]. Journal of Geophysical Research: Oceans, 119 (3): 1593-1608

Galbraith H, Jones R, Park RA, et al, editors. 2003. Ecological forecasting: New tools for coastal and marine ecosystem management [M]. Silver Spring, Maryland: NOAA.

Hall J W, Meadowcroft I C, Sayers P B, et al. 2003. Integrated flood risk management in England and Wales [J]. Natural Hazards Review, 4(3): 126-135.

Hallegatte S, Green C, Nicholls R J, et al. 2013. Future flood losses in major coastal cities [J]. Nature Climate Change, 3 (9): 802-806.

Hanson S, Nicholls R, Ranger N, et al. 2011. A global ranking of port cities with high exposure to climate extremes [J]. Climatic Change, 104(1): 89-111.

Hinkel J, Klein R J T. 2009. Integrating knowledge to assess coastal vulnerability to sea-level rise: The development of the DIVA tool [J]. Global Environmental Change, 19(3): 384-395.

Hinkel J, Lincke D, Vafeidis A T, et al. 2014. Coastal flood damage and adaptation costs under 21 st century sea-level rise [J]. PNAS, 111(9): 3292-3297.

Hinkel J, Jaeger C, Nicholls R J, et al. 2015. Sea-level rise scenarios and coastal risk management $[\mathrm{J}]$. Nature Climate Change, 5(3): 188-190.

Hoozemans F M J, Marchand M, Pennekamp H A. 1993. Sea level rise: A global vulnerability assessment: Vulnerability assessment for population, coastal wetlands and rice production on a global scale $[\mathrm{M}]$. Hague, the Netherlands: Delft Hydraulics.

Hu P, Zhang Q, Shi P, et al. 2018. Flood-induced mortality across the globe: Spatiotemporal pattern and influencing factors $[\mathrm{J}]$. Science of the Total Environment, 643: 171182.

IPCC. 1990. Intergovernmental panel on climate change climate change 1990: First assessment report (AR1) [M]. Cambridge, UK: Cambridge University Press.

IPCC. 2013. Intergovernmental panel on climate change climate change 2013: Fifth assessment report (AR5) [M]. Cambridge, UK: Cambridge University Press.

Jevrejeva S, Grinsted A, Moore J C. 2014. Upper limit for sea level projections by 2100 [J]. Environmental Research Letters, 9, doi: 10.1088/1748-9326/9/10/104008.

Jongman B, Ward P J, Aerts J C J H. 2012. Global exposure to river and coastal flooding: Long term trends and changes [J]. Global Environmental Change, 22(4): 823-835.

Jonkman S N, Vrijling J K. 2008. Loss of life due to floods [J].
Journal of Flood Risk Management, 1(1): 43-56.

Kang L, Ma L, Liu Y. 2016. Evaluation of farmland losses from sea level rise and storm surges in the Pearl River Delta region under global climate change $[\mathrm{J}]$. Journal of Geographical Sciences, 26(4): 439-456.

Kebede A S, Nicholls R J. 2012. Exposure and vulnerability to climate extremes: Population and asset exposure to coastal flooding in Dar es Salaam, Tanzania [J]. Regional Environmental Change, 12(1): 81-94.

Klein R J T, Nicholls R J. 1999. Assessment of coastal vulnerability to climate change [J]. AMBIO, 28(2): 182-187.

Kopp R E, Horton R M, Little C M, et al. 2014. Probabilistic 21 st and 22nd century sea-level projections at a global network of tide-gauge sites [J]. Earth's Future, 2(8): 383-406.

Lamb R, Keef C, Tawn J, et al. 2010. A new method to assess the risk of local and widespread flooding on rivers and coasts [J]. Journal of Flood Risk Management, 3(4): 323-336.

Lemmen D S, Warren F J, James T S, et al. 2016. Canada's marine coasts in a changing climate [R]. Ottawa, Canada: Government of Canada.

Lin N, Emanuel K, Oppenheimer M, et al. 2012. Physically based assessment of hurricane surge threat under climate change [J]. Nature Climate Change, 2(6): 462-467.

Lin N, Emanuel K. 2016. Grey swan tropical cyclones [J]. Nature Climate Change, 6(1): 106-111.

Linham M M, Nicholls R J. 2012. Adaptation technologies for coastal erosion and flooding: A review [J]. Proceedings of the ICE-Maritime Engineering, 165(3): 95-112.

Liu J, Wen J, Huang Y, et al. 2015. Human settlement and regional development in the context of climate change: A spatial analysis of low elevation coastal zones in China $[\mathrm{J}]$. Mitigation and Adaptation Strategies for Global Change, 20 (4): $527-546$

Lowe J, Howard T, Pardaens A, et al. 2009. UK Climate Projections science report: Marine and coastal projections [M]. Exeter, UK: Met Office Hadley Centre.

Ma Z, Melville D S, Liu J, et al. 2014. Rethinking China's new great wall [J]. Science, 346: 912-914.

Marcos M, Tsimplis M N, Shaw A G P. 2009. Sea level extremes in southern Europe [J]. Journal of Geophysical Research: Oceans, 114, doi: 10.1029/2008JC004912.

Mawdsley R J, Haigh I D. 2016. Spatial and temporal variability and long-term trends in skew surges globally [J]. Frontiers in Marine Science, 3. doi: 10.3389/fmars.2016.00029.

Mcleod E, Poulter B, Hinkel J, et al. 2010. Sea-level rise impact models and environmental conservation: A review of models and their applications [J]. Ocean \& Coastal Management, 53(9): 507-517. 
Menéndez M, Woodworth P L. 2010. Changes in extreme high water levels based on a quasi-global tide-gauge data set [J]. Journal of Geophysical Research: Oceans, 115. doi: 10.1029/2009JC005997.

Muis S, Verlaan M, Winsemius H C, et al. 2016. A global reanalysis of storm surges and extreme sea levels [J]. Nature Communications, 7. doi: 10.1038/ncomms11969.

Nicholls R J. 2004. Coastal flooding and wetland loss in the 21st century: Changes under the SRES climate and socioeconomic scenarios [J]. Global Environmental Change, 14 (1): 69-86.

Nicholls R J, Cazenave A. 2010. Sea-level rise and its impact on coastal zones [J]. Science, 328: 1517-1520.

Nicholls R J, Hanson S E, Lowe J A, et al. 2014. Sea-level scenarios for evaluating coastal impacts $[\mathrm{J}]$. Wiley Interdisciplinary Reviews: Climate Change, 5(1): 129-150.

Nicholls R J, Hoozemans F M J, Marchand M. 1999. Increasing flood risk and wetland losses due to global sea-level rise: Regional and global analyses [J]. Global Environmental Change, 9: S69-S87.

Nicholls R J, Mimura N. 1998. Regional issues raised by sealevel rise and their policy implications $[\mathrm{J}]$. Climate Research, 11(1): 5-18.

Parris A S, Bromirski P, Burkett V, et al. 2012. Global sea level rise scenarios for the United States National Climate Assessment [R]. NOAA Tech Memo OAR Climate Program Office.

Pelling M, Blackburn S. 2014. Megacities and the coast: Risk, resilience and transformation [M]. London, UK: Routledge.

Rahmstorf S. 2017. Rising hazard of storm-surge flooding [J]. PNAS, 114(45): 11806-11808.

Rosenzweig C, Solecki W D. 2010. Introduction to climate change adaptation in New York City: Building a risk management response [J]. Annals of the New York Academy of Sciences, 1196: 1-17.

Rowley R J, Kostelnick J C, Braaten D, et al. 2007. Risk of rising sea level to population and land area $[\mathrm{J}]$. Eos, Transactions, American Geophysical Union, 88(9): 105-107.

Sayers P B, Horritt M, Penning-Rowsell E, et al. 2017. Climate change risk assessment 2017: Projections of future flood risk in the UK [R]. London, UK: Committee on Climate Change.

Shibayama T. 2015. Field surveys of recent storm surge disasters [J]. Procedia Engineering, 116: 179-186.

Spencer T, Schuerch M, Nicholls R J, et al. 2016. Global coastal wetland change under sea-level rise and related stresses: The DIVA wetland change model [J]. Global and Planetary Change, 139: 15-30.

Sterr H. 2008. Assessment of vulnerability and adaptation to sea-level rise for the coastal zone of Germany $[\mathrm{J}]$. Journal of Coastal Research, 24(2): 380-393.

Su S, Pi J, Wan C, et al. 2015. Categorizing social vulnerability patterns in Chinese coastal cities $[\mathrm{J}]$. Ocean \& Coastal Management, 116: 1-8.

Syvitski J P M, Kettner A J, Overeem I, et al. 2009. Sinking deltas due to human activities [J]. Nature Geoscience, 2 (10): 681-686.

Townsend. 2006. The federal response to hurricane katrina-lessons learned [R]. Washington, DC: White House.

UNISDR. 2009. Terminology on disaster risk reduction [R]. New York, NY: UNISDR.

Vousdoukas M I, Mentaschi L, Voukouvalas E, et al. 2018. Climatic and socioeconomic controls of future coastal flood risk in Europe [J]. Nature Climate Change, 8(9): 776-780.

Wahl T, Chambers D P. 2015. Evidence for multidecadal variability in US extreme sea level records [J]. Journal of Geophysical Research: Oceans, 120(3): 1527-1544.

Wahl T, Haigh I D, Nicholls R J, et al. 2017. Understanding extreme sea levels for broad-scale coastal impact and adaptation analysis [J]. Nature Communications, 8. doi: 10.1038/ ncomms 16075 .

Wahl T, Jain S, Bender J, et al. 2015. Increasing risk of compound flooding from storm surge and rainfall for major US cities [J]. Nature Climate Change, 5(12): 1093-1097.

Wang J, Gao W, Xu S, et al. 2012. Evaluation of the combined risk of sea level rise, land subsidence, and storm surges on the coastal areas of Shanghai, China [J]. Climatic Change, 115(3-4): 537-558.

Ward P J, Jongman B, Aerts J C J H, et al. 2017. A global framework for future costs and benefits of river-flood protection in urban areas [J]. Nature Climate Change, 7(9): 642-646.

Willis H H, Narayanan A, Fischbach J R, et al. 2016. Current and future exposure of infrastructure in the United States to Natural Hazards [M]. California, CA: RAND.

Woodruff J D, Irish J L, Camargo S J. 2013. Coastal flooding by tropical cyclones and sea-level rise [J]. Nature, 504: 4452.

Woodworth P L, Blackman D L. 2004. Evidence for systematic changes in extreme high waters since the mid-1970s [J]. Journal of Climate, 17(6): 1190-1197.

Woodworth P L, Menéndez M, Gehrels W R. 2011. Evidence for century-timescale acceleration in mean sea levels and for recent changes in extreme sea levels [J]. Surveys in Geophysics, 32(4-5): 603-618.

Wu S, Feng A, Gao J, et al. 2017. Shortening the recurrence periods of extreme water levels under future sea-level rise 
[J]. Stochastic Environmental Research and Risk Assessment, 31(10): 2573-2584.

Yin J, Yin Z, Xu S. 2013. Composite risk assessment of typhoon-induced disaster for China's coastal area [J]. Natural Hazards, 69(3): 1423-1434.

Yin J, Yu D, Lin N, et al. 2017. Evaluating the cascading impacts of sea level rise and coastal flooding on emergency response spatial accessibility in Lower Manhattan, New York City [J]. Journal of Hydrology, 555: 648-658.

Yin J, Yu D, Yin Z, et al. 2016. Evaluating the impact and risk of pluvial flash flood on intra-urban road network: A case study in the city center of Shanghai, China [J]. Journal of Hydrology, 537: 138-145.

Yu D, Lane S N. 2006a. Urban fluvial flood modelling using a two- dimensional diffusion- wave treatment, part 1: Mesh resolution effects [J]. Hydrological Processes, 20(7): 15411565.

Yu D, Lane S N. 2006b. Urban fluvial flood modelling using a two-dimensional diffusion-wave treatment, part 2: Development of a sub-grid-scale treatment [J]. Hydrological Processes, 20(7): 1567-1583.

Zheng F F, Westra S, Leonard M, et al. 2014. Modeling dependence between extreme rainfall and storm surge to estimate coastal flooding risk [J]. Water Resources Research, 50(3): 2050-2071.

Zscheischler J, Westra S, Hurk B J, et al. 2018. Future climate risk from compound events $[\mathrm{J}]$. Nature Climate Change, 8 : 469-477.

\title{
A review of coastal flood risk research under global climate change
}

\author{
FANG Jiayi ${ }^{1,2,3}$, SHI Peijun ${ }^{2,3,45^{*}}$ \\ (1. Key Laboratory of Geographic Information Science, Ministry of Education, School of Geographic Sciences, \\ East China Normal University, Shanghai 200241, China; 2. Faculty of Geographical Science, Beijing Normal \\ University, Beijing 100875, China; 3. Academy of Disaster Reduction and Emergency Management, Ministry of \\ Emergency Management \& Ministry of Education, Beijing 100875, China; 4. State Key Laboratory of Earth \\ Surface Processes and Resource Ecology, Beijing Normal University, Beijing 100875, China; \\ 5. School of Geographical Science, Qinghai Normal University, Xining 810016, China)
}

\begin{abstract}
The sea level rise under global climate change and coastal floods caused by extreme sea levels due to the high tide levels and storm surges have huge impacts on coastal society, economy, and natural environment. It has drawn great attention from global scientific researchers. This study examines the definitions and elements of coastal flooding in the general and narrow senses, and mainly focuses on the components of coastal flooding in the narrow sense. Based on the natural disaster system theory, the review systematically summarizes the progress of coastal flood research in China, then discusses existing problems in present studies and future research directions with regard to this issue. It is proposed that future studies need to strengthen research on adapting to climate change in coastal areas, including studies on the risk of multi- hazards and uncertainties of hazard impacts under climate change, risk assessment of key exposure (critical infrastructure) in coastal hotspots, and cost-benefit analysis of adaptation and mitigation measures in coastal areas. Efforts to improve the resilience of coastal areas under climate change should be given more attention. The research community also should establish the mechanism of data sharing among disciplines to meet the needs of future risk assessments, so that coastal issues can be more comprehensively, systematically, and dynamically studied.
\end{abstract}

Keywords: coastal flood; global climate change; storm surge; risk assessment; impact 